\title{
Retracted: Research on Wushu Sports Feedback System Based on Real-Time Target Intelligent Tracking Video Processing
}

\author{
Security and Communication Networks \\ Received 15 November 2022; Accepted 15 November 2022; Published 8 December 2022 \\ Copyright ( 2022 Security and Communication Networks. This is an open access article distributed under the Creative Commons \\ Attribution License, which permits unrestricted use, distribution, and reproduction in any medium, provided the original work is \\ properly cited.
}

Security and Communication Networks has retracted the article titled "Research on Wushu Sports Feedback System Based on Real-Time Target Intelligent Tracking Video Processing" [1] due to concerns that the peer review process has been compromised.

Following an investigation conducted by the Hindawi Research Integrity team [2], significant concerns were identified with the peer reviewers assigned to this article; the investigation has concluded that the peer review process was compromised. We therefore can no longer trust the peer review process, and the article is being retracted with the agreement of the editorial board.

\section{References}

[1] X. Liu, J. Liu, J. Fan, and X. Li, "Research on Wushu Sports Feedback System Based on Real-Time Target Intelligent Tracking Video Processing," Security and Communication Networks, vol. 2022, Article ID 4599005, 10 pages, 2022.

[2] L Ferguson, "Advancing Research Integrity Collaboratively and with Vigour," 2022, https://www.hindawi.com/post/advancingresearch-integrity-collaboratively-and-vigour/. 


\title{
Research on Wushu Sports Feedback System Based on Real-Time Target Intelligent Tracking Video Processing
}

\author{
Xianfa Liu, ${ }^{1}$ Jianfang Liu $\mathbb{D}^{2},{ }^{2}$ Jiangtao Fan, ${ }^{3}$ and Xing $\mathrm{Li}^{4}$ \\ ${ }^{1}$ China Wushu College, Beijing Sport University, Beijing 100084, China \\ ${ }^{2}$ Institute of Physical Education, NanChang JiaoTong Institute, Nan'chang, Jiang'xi, China \\ ${ }^{3}$ School of Taichi Culture, Handan University, Handan 056000, Hebei, China \\ ${ }^{4}$ Institute of Physical Education, Handan University, Handan 056000, Hebei, China
}

Correspondence should be addressed to Jianfang Liu; 2019020106@stu.cdut.edu.cn

Received 8 January 2022; Revised 17 January 2022; Accepted 8 February 2022; Published 26 February 2022

Academic Editor: Muhammad Arif

Copyright $(\odot 2022$ Xianfa Liu et al. This is an open access article distributed under the Creative Commons Attribution License, which permits unrestricted use, distribution, and reproduction in any medium, provided the original work is properly cited.

In order to better improve the martial arts learning effect, this paper puts forward the design method of martial arts motion feedback system based on target intelligent tracking video processing, optimizes the hardware configuration of the system, adds a composite tracker to the system, so as to effectively track the motion changes of different human targets, and further combines the real-time target intelligent tracking video processing technology and builds a moving target tracking model; then, based on the calculation of motion characteristic parameters, CRF method is used for motion behavior recognition and feedback. Finally, simulation studies reveal that a martial arts motion feedback system based on real-time target intelligent tracking video processing can track moving targets more accurately and has the potential for practical implementation.

\section{Introduction}

To some degree, Sports Wushu video teaching may be thought of as a process in which instructors give students appropriate learning material, feedback, and the ability to change the practice process at any moment in order to increase action level and performance [1]. To make teaching more effective, we must provide students with two types of information to correct errors: first, how does the completed operation compare with the required operation? Second, how can you change the incorrect operation to make it closer to the requirements? Both types of information are called feedback [2]. Feedback is the general term of task internal feedback and additional feedback, which refers to the information about motor skill operation obtained by individuals during or after practice. Additional feedback plays two roles in the process of motor skill learning. The first is to promote the acquisition of action goals. Because additional feedback provides information about the success of skill operation, learners can determine the appropriate activity content in order to operate the skills correctly [3]. In this way, compared with not obtaining any external information, additional feedback can help individuals achieve skill goals faster and easier. The second function is to motivate learners to make continuous efforts towards their goals. At this time, the operator compares his operation with the target performance through additional feedback, and then the individual must decide whether to continue to work towards the target, change the target, or stop the operation activities.

\section{Real-Time Target Intelligent Tracking Video Wushu Motion Feedback System}

2.1. System Hardware Structure Configuration. According to the functional requirements of martial arts video analysis system and drawing on the previous research results, in order to realize the cross platform transplantation and extended application of the system, the system is established on the computer platform, using the visual studio 2018 development environment integrating multiple programming languages and supported by $\mathrm{C}++$ development language. Moreover, MFC encapsulates the windows API in the form 
of $\mathrm{C}++$ class to build a basic class library for application development, which can simplify system development. Therefore, under the MFC framework, combined with MySQL database, opencv open-source cross platform computer vision library is selected to support the processing of video image sequence information [4]. The development of video analysis system is realized under the environment of windows and Linux. Tms320c6678 processor with 8 DSP cores is selected. Its maximum running speed can reach $1.25 \mathrm{GHz}$. It is configured with a double-click buffer architecture of $512 \mathrm{~KB}$ L2 memory. The storage space and digital signal processing area contain a primary buffer architecture, respectively [5]. Among them, the storage space buffer architecture can quickly store martial arts video. On this basis, the digital signal processing area buffer architecture can map the moving video to other memories. This synchronous dynamic random access memory can provide an alternative storage scheme for the system in case of abnormal failure of the digital processor. At the same time, the system also includes video image data acquisition, power module, universal asynchronous transceiver control terminal, network control chip, storage bus, and JTAG [6]. After acquiring the martial arts video, the system will use the storage bus to allocate the storage address and carry out functional storage through the digital processor and components. The network control chip can regulate the system network bandwidth. JTAG is used to adjust and test the status of the digital processor [7]. The power module provides $5.5 \mathrm{~V}, 3.5 \mathrm{~V}$, and $2.0 \mathrm{~V}$ power supply for video acquisition, digital processor, and storage bus, respectively. The hardware framework of Wushu video analysis system is shown in Figure 1.

The motion feedback system ensures the accuracy of probe motion in $X$ and $Y$ planes. Due to the existence of friction, speed, and other factors, the probe will produce motion errors when it moves frequently in the $X$ and $y$-axis planes. The device drivers of intelligent target tracking video system are generally divided into three categories: character device driver, block device driver, and network device driver [8]. Character device is the most commonly used device for target intelligent tracking video. It must access the device in the form of byte stream (file), such as keyboard, mouse, touch screen, and printer. The information on the block device is organised into blocks that may be accessed in any order. Hard discs, U discs, flash drives, and other devices all have a defined address. Character and block equipment are not the same as network equipment. At the same time, it has certain qualities of both character and block equipment. The socket interface [9] is used to implement it. It does not relate to a file system node and may transmit and receive data in data stream and datagram formats. Bluetooth, mouse, USB drive, SATA driver, RTC driver, and other common device drivers have been incorporated into the target intelligent tracking video system. However, due to the wide update speed and variety of chips, not all devices can find corresponding drivers in the target intelligent tracking video [10], for example, the video acquisition chip tw2835, serial port expansion chip sc16is752, IO port expansion chip pca9635, and other chips in the intelligent monitoring system designed in this paper. In order to support the above devices under the target intelligent tracking video, the corresponding driver must be designed according to the structure of the target intelligent tracking video driver and the functional requirements of the intelligent monitoring system. The drivers to be developed in this article are shown in Table 1.

Although the position information of the probe can be obtained by using the encoder on the servo motor, it can only form a semi-closed-loop position control system because it can not overcome the transmission error between various parts of the system and the error can not be compensated [11]. This research decides to install high-precision position feedback components on the final moving part to collect the real position information of the probe in order to eliminate mechanical faults and construct a comprehensive closed-loop control system in order to achieve better control accuracy. The block diagram of the full closed-loop position control system is shown in Figure 2 below.

The platform obtains the target coordinates from the PC end. After the platform probe moves to the target coordinates, the position feedback element detects the actual coordinates of the probe at this time [12]. The feedback system compares the actual coordinates with the target coordinates. If the difference between the two is greater than the threshold, error correction is required. Adjust the error to obtain the correction distance that the probe should move. After the probe moves the corresponding distance, the position feedback element detects the actual coordinates of the probe again [13]. If the difference between the two is less than the threshold at this time, the correction process ends; otherwise repeat the above process.

\subsection{System Software Function and Process. Based on the real-} time monitoring of moving targets, the martial arts video analysis system will collect the video image sequence of sports and analyze the strategy and tactics, training methods, and scoring accuracy in martial arts through moving target detection, tracking, and behavior recognition and understanding, so as to realize the "intelligence" of video assisted training feedback and avoid misjudgment [14]. The software is under the windows operating system. The development mode of combining platform and professional software is adopted. This software is installed and used under Windows $\mathrm{XP} / \mathrm{Vista}$ operating system. Thus, the functional flow of the system is shown in Figure 3 below.

Motion segmentation includes motion detection and target classification. The purpose of motion detection is to extract the changed region from the background image from the sequence image. The effective segmentation of moving region is very important for postprocessing such as target classification, tracking, and behavior understanding, because the subsequent processing only considers the pixels corresponding to the moving region in the image. The postprocessing of motion detection is target classification [15]. The goal of target classification is to extract the motion area that corresponds to a person from the motion region that has been observed. The precise categorization of moving objects 


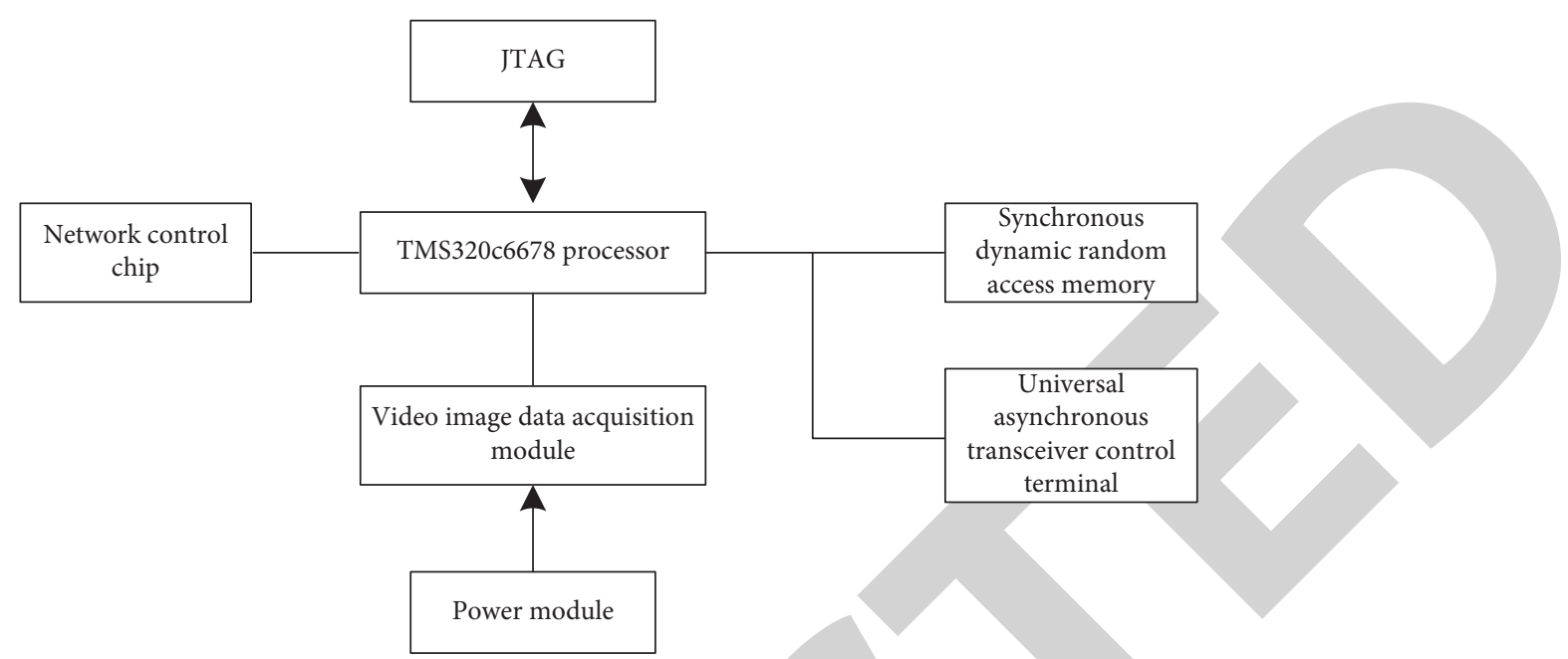

FIgURE 1: Hardware framework of Wushu sports video analysis system.

is critically important in order to permit subsequent pedestrian monitoring and behavior analysis. The proposed video based target intelligent tracking human motion generating technique uses a field-based target intelligent tracking motion database and extracts the target intelligent tracking motion information's technical route from 2D contour data [16]. This method can only be used for motion reconstruction of specific motion types because it depends on the target intelligent tracking motion database of specific domain knowledge. But at the same time, it is a general system framework: the type of motion database determines the range of applicable motion types. The main research contents of this paper include target intelligent tracking attitude reconstruction based on contour matching; intelligent target tracking attitude refinement based on video content; human motion reconstruction combined with spatiotemporal model of motion library [17]. On this basis, a video based $3 \mathrm{D}$ human motion generation platform $\mathrm{V}$ hsportstrackingver 1.0 is realized. The overall flow of video based intelligent target tracking motion generation is shown in Figure 4.

Firstly, the video analysis system collects the video image data of the moving target and imports it into the system, extracts the required moving target from the background image of the video sequence, and tracks it according to the speed, position, color, texture, and other information of the target in the continuous image frame sequence [18]. Then, based on the classification and matching of the motion feature data, calculate the characteristic parameters of each node of the moving target to identify the motion behavior mode and state. So far, the intelligent analysis of the whole video image sequence is completed. The basic composition of the platform mainly includes video acquisition unit, which can use any equipment with video recording function such as camera, camera, mobile phone, and camera; a video processing unit, including a computer and corresponding software; storage unit, where computer hard disk can be used, preferably a relatively independent hard disk. The display unit and the display device receive the results sent by the computer and feed back to the practitioner in the form of video. It may be the computer's display screen, ideally an independent flat-panel TV or projector with a screen greater than 32 inches. In the preprocessing stage, there are many processing stages. It primarily conducts different actions on the input video data during the motion reconstruction step [19]. It mainly includes human contour extraction (motion segmentation) in video, human pose reconstruction based on video, pose deformation refinement based on video content, target intelligent tracking motion generation based on statistical modeling of motion library, etc. The processing content in the motion reconstruction stage is shown in Figure 5.

The position prediction of moving target is realized by Kalman filter. In order to track the position of moving objects in video sequence, Kalman filter needs to be designed for moving objects, and Kalman filter is used to estimate the position of moving objects in other graphics. In the video sequence, the moving object's position in the adjacent frame images changes slowly, so the moving object's motion can be assumed to be a uniform linear motion, and the moving target area can be marked with a rectangular frame in the moving image tracking. The motion formula is shown in the formula.

$$
s_{k}=s_{k-1}+(\Delta t) v_{k-1} .
$$

Among them:

$$
v_{k}=v_{k-1} \text {, }
$$

where $s_{k}=[x(k), y(k)], \mathrm{y}(\mathrm{k})$ is the position of the moving target, $t$ is the time interval, and $v k$ is the speed of the moving target. At this time, the state vector of Kalman filter is taken as

$$
L_{k}=v_{k}[x(k), y(k)]^{T}-s_{k} .
$$

Target intelligent tracking motion database is the basis of video based human motion generation technology in this paper. It is not only a data source for intelligent target 
TABLE 1: List of drivers to be developed.

\begin{tabular}{lc}
\hline Drive name & Drive function introduction \\
\hline TW2835 & Be responsible for collecting analog video signals and converting them into digital video signals \\
SC16IS752 & $12 \mathrm{C}$ bus expands 3 serial ports \\
PCA9635 & $12 \mathrm{C}$ bus extends 16 GPIO ports for panel control \\
GPIO & Be responsible for the space-time relay and read the sensor status \\
DS1340 & Provide real-time clock \\
\hline
\end{tabular}

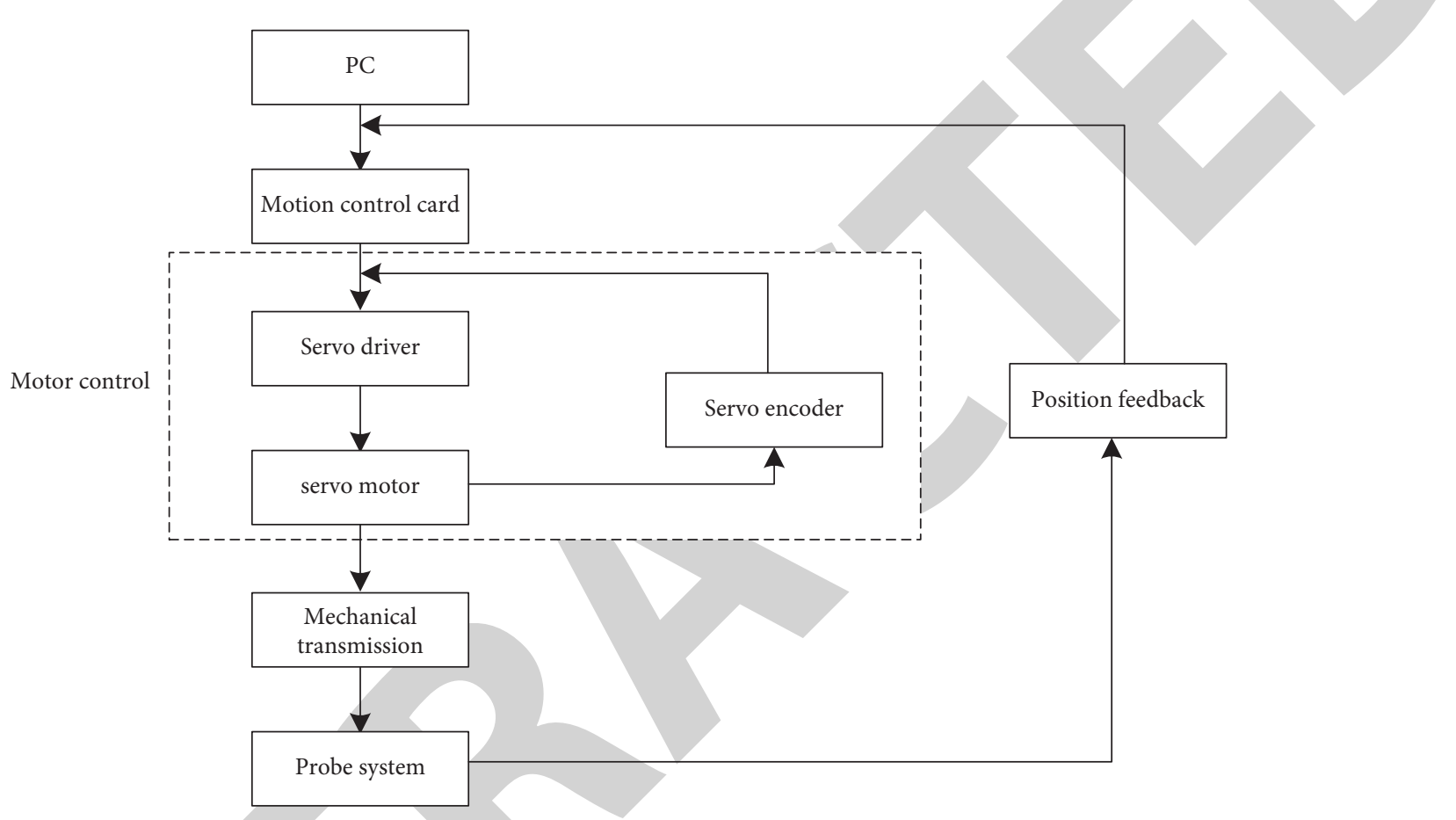

FIgURE 2: Functional structure of full closed-loop control module of system hardware equipment.

tracking attitude reconstruction based on video, but also a data source for intelligent target tracking attitude reconstruction based on video. Furthermore, according to the motion database, the spatiotemporal models (spatial structure similarity model and Markov time probability transfer model) provide important guiding information for human motion reconstruction, which is an important basis for automatic continuous motion generation in this paper [20]. Therefore, how to establish such a target intelligent tracking human motion database is very important [21].

2.3. Realization of Wushu Sports Feedback. When building the moving target tracking model, multiple trackers are used to collect the data in the martial arts video from multiple perspectives. The target tracker of the basic motion model tracks high-speed and ordinary moving targets, respectively. The mathematical expression of the model is

$$
p_{j}\left(X_{t} \mid X_{t-1}\right)=L_{k}+G\left(X_{t-1}, \sigma_{3}^{2}\right) \text {. }
$$

$\mathrm{Xt}$ is the moving target behavior data at time $t, \mathrm{j}$ is the target tracker sequence, and $G$ is the normal distribution function, $G\left(X_{t-1}, \sigma_{j}^{2}\right)$ is a target, the behavior data is $x_{t-1}$, and the behavior variance is $\sigma_{2 \mathrm{j}}$. The core of the behavior observation model is to create a Markov chain on the above basic motion model, which needs to first set the behavior direction of a moving target and verify the feasibility of the direction. The mathematical expression of the model is

$$
Q_{j}\left(X_{t}^{j *} \mid X_{t}^{j}\right)=p_{j}\left(X_{t}^{j *} \mid X_{i}^{j}\right),
$$

where $Q_{j}$ is the direction setting function of the moving target behavior, and $x_{j}, x_{\mathrm{t}}$ is the direction set by the basic motion state $\mathrm{x}_{\mathrm{jt}}$ of the $\mathrm{jth}$ target tracker at time $t$. The set direction verification function of the moving target behavior observation model is

$$
\gamma=\min \left[\frac{p_{j}\left(X_{t} \mid X_{t}^{j *}\right) Q_{j}\left(X_{t}^{j} \mid X_{t}^{j *}\right)}{p_{j}\left(X_{t} \mid X_{t}^{j}\right) Q_{j}\left(X_{t}^{j *} \mid X_{t}^{j}\right)}\right],
$$

where min [] is the minimum value obtained within a specific range, and set $\gamma$ is threshold. When the above formula reaches the threshold, the set martial arts video analysis direction can be used as the output result. Imcmc tracking algorithm has two kinds of sampling modes: parallel and interactive. In the parallel mode, each Markov chain samples independently. Set time $t$, the current state of the basic motion tracker $t$ is $x$, and propose a new state $X$ by using the formula; then the probability of $X^{*}$ being received is 


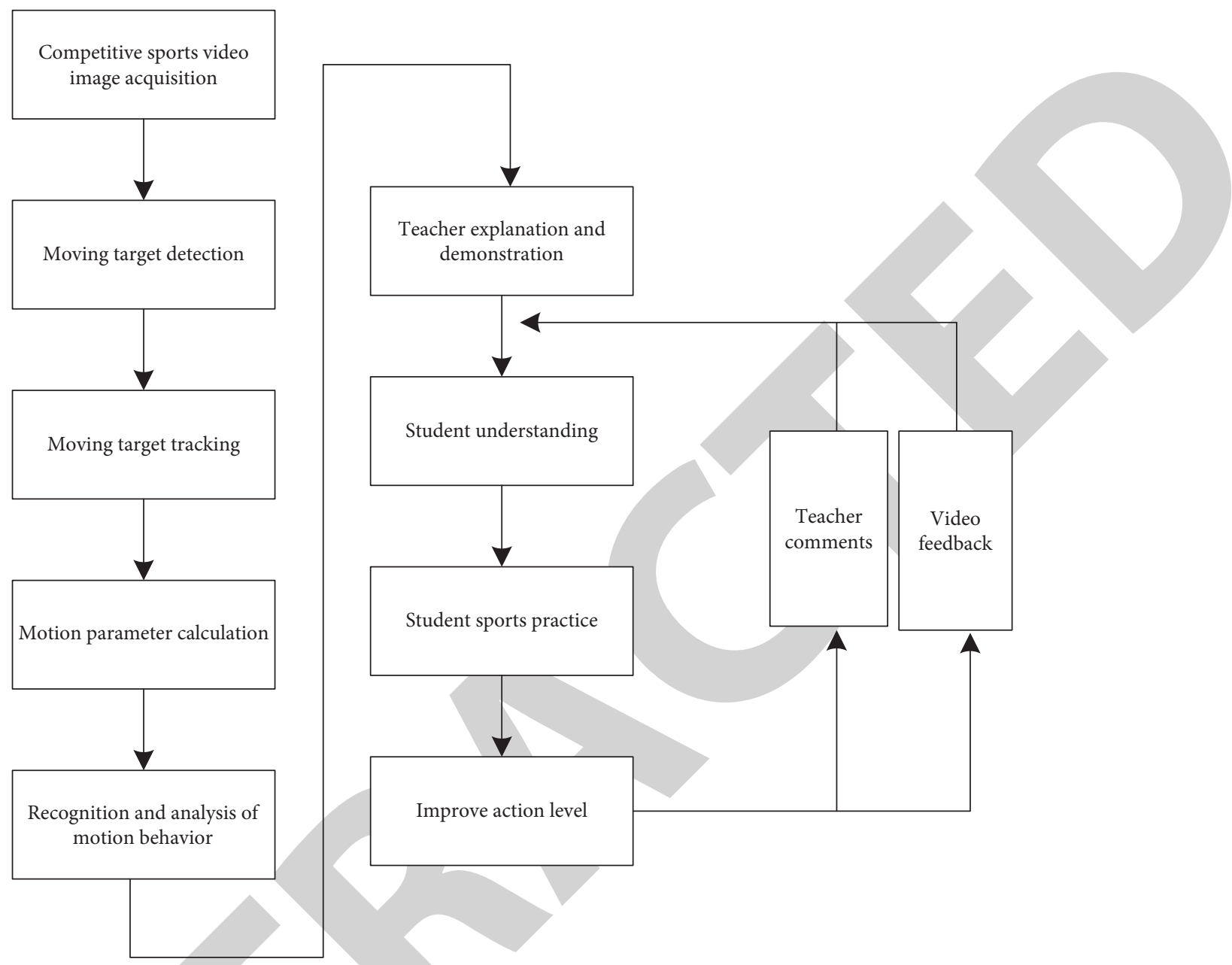

FIGURE 3: Data processing function model of Wushu video analysis system.

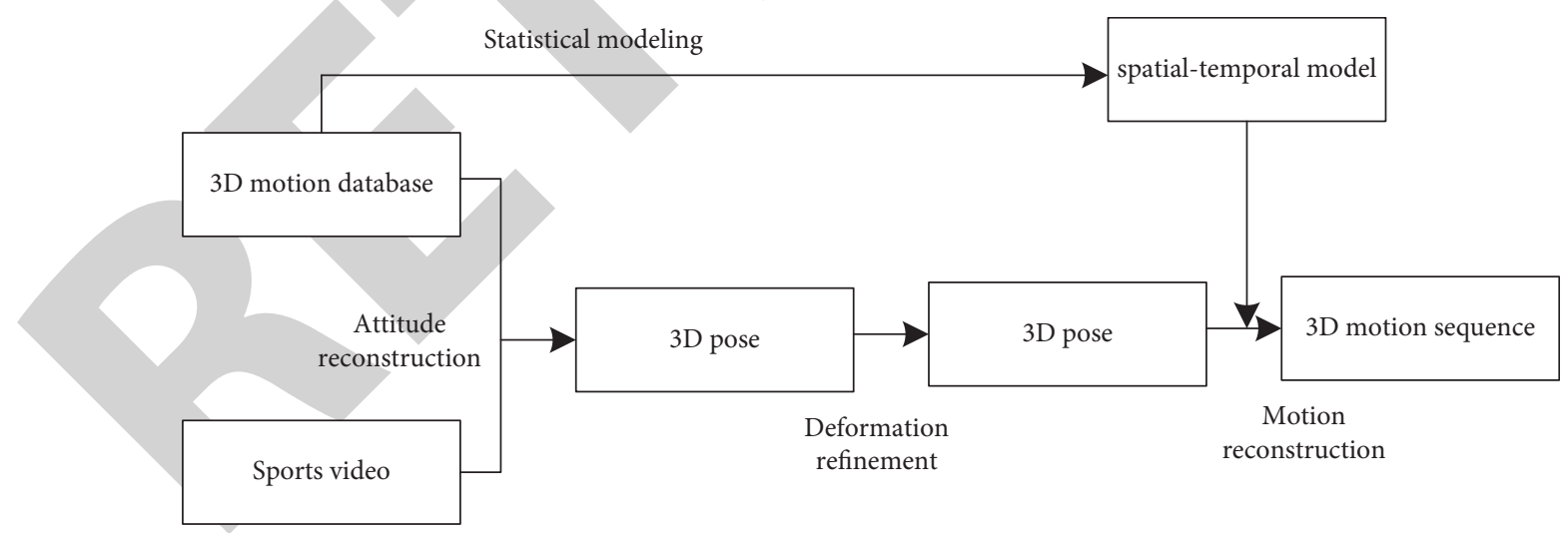

FIgURE 4: Overall technical framework of video based motion generation.

$$
\partial_{\text {patallel }}=\min \left[1, \frac{p_{j}\left(Y_{t} \mid X_{t}^{j *}\right) Q_{i}\left(X_{i}^{j} ; X_{1}^{j *}\right)}{p_{j}\left(Y_{1} \mid X_{t}^{j}\right) Q_{i}\left(X_{t}^{j *} ; X_{t}^{j}\right)}\right],
$$

where $p(y \mid x)$ is the possible value of the ith observation model obtained through the jth motion model. In the interactive mode, the trackers interact with each other without proposing a function to propose a new state. The key is to consider whether to receive the states recommended by other trackers. In the construction of Markov chain, the tracking algorithm sets a limit for compatibility with parallel and interactive modes $\gamma$. To automatically switch between the two modes, set that each Markov chain should sample $n$ particles, and Y is initially 1 . The construction process of Markov chain is as follows: use rand() function to generate a random number between $[0,1]$; if the $\operatorname{rand}()$ function is not satisfied $\gamma$, then the interaction mode is 


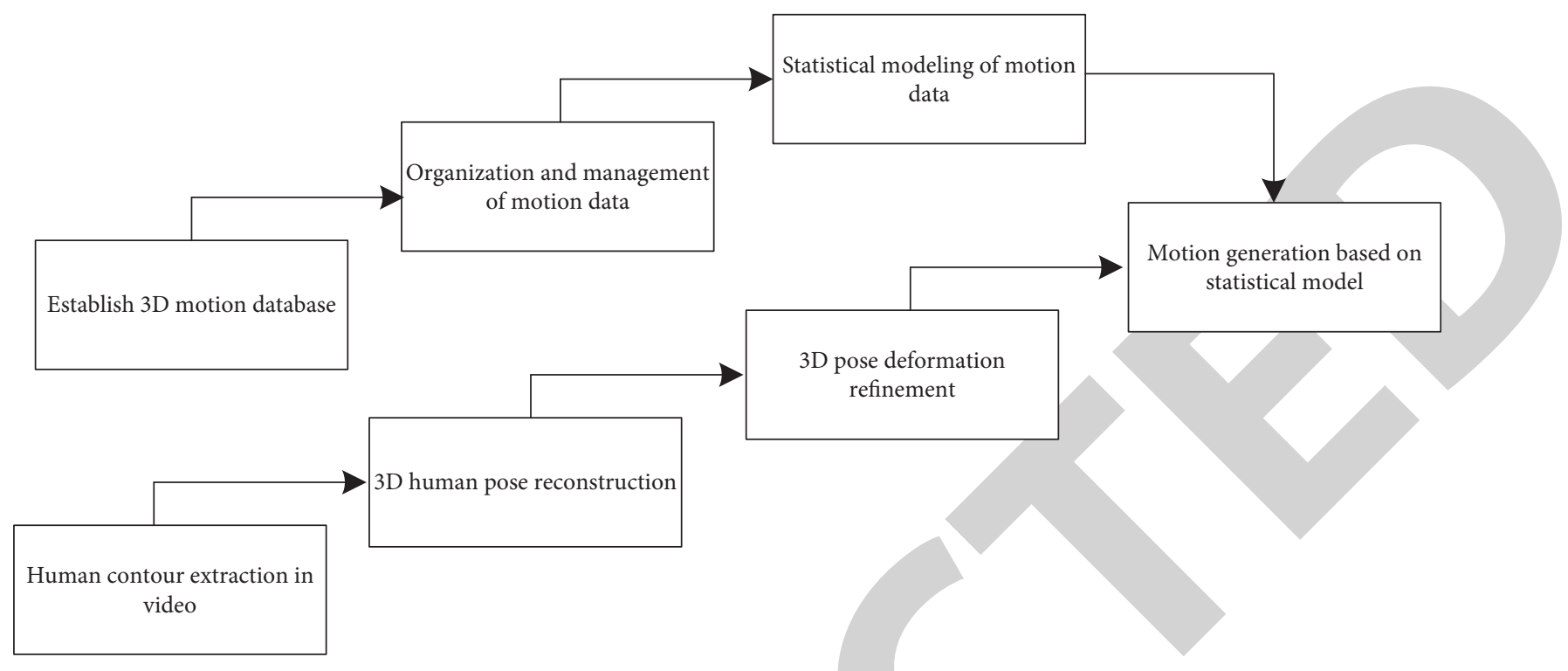

Figure 5: Process flow of pretreatment stage.

selected, and the calculated probability receives the new state as the state of this Markov chain; if the rand() function satisfies $\gamma$, then the formula is used to propose a new state, and the formula is used to calculate the reception probability of the new state. The $y$ value in the formula decreases from 1 to 0.5 in the cycle. Imcmo moving target tracking algorithm uses the construction of parallel Markov chains with different observation and motion models to achieve the purpose of building a tracking model based on composite tracker. In the parallel mode, information interaction can be carried out between Markov chains, which can timely correct the deviation of individual tracker samples from the construction direction of Markov chain and optimize the quality of moving target video acquisition to achieve better tracking effect. According to the above three-dimensional coordinate reconstruction results, the parameters are measured. The method of minimizing the projection error of image sequence is used to obtain the motion parameters and structural parameter matrix in the process of athletes' three-dimensional motion and finally complete the accurate analysis of three-dimensional motion parameters. Firstly, it is assumed that the matrix formula of the measured athlete characteristic points is $\mathrm{m}_{x y}$; the moving image feature point matrix to be predicted is $\mathrm{m}_{x y}$. If the feature points of each moving image are $\left[\begin{array}{l}t_{x y} \\ e_{x y}\end{array}\right], i=1, \ldots, J, c=1, \ldots, Q$, the measurement matrix is $\mathrm{m}_{x y}$, where $j$ represents the number of moving image frames and $Q$ represents the number of feature points of single frame moving image. The ultimate goal of the matrix is to obtain the three-dimensional structure of each frame moving image. If the three-dimensional shape of an athlete's joint is a weighted linear set of shape bases, there are

$$
F=\partial_{\text {parallel }} \sum_{i=1}^{N} \delta_{x y} \lambda_{x}
$$

In the formula: $\delta_{x y}$ represents the weighting coefficient, $\lambda_{x}$ represents the shape base, and $N$ represents the number of shape bases. If $N=1, \delta_{x y}$ is the state of the key area under the weak perspective projection model is

$$
m_{x y}=\left[\begin{array}{c}
t_{x 1}, \ldots, t_{x Q} \\
e_{x 1}, \ldots, e_{x Q}
\end{array}\right]=\bar{w}\left(\sum_{i=1}^{N} \delta_{x y} \lambda_{x}\right)+F \bar{D}_{x} k_{v}^{D} .
$$

In the formula: $\bar{w}$ represents the first two rows of the rotation matrix, and $\bar{D}_{x}$ represents the first two elements of the translation vector. In order to optimize the operation process of the algorithm, the coordinates of each moving feature image shall be changed to ensure that the origin of the moving image coordinate system is at the center of the image point, and then the translation vector shall be filtered. The process is shown in the formula:

$$
\left\{\bar{t}_{x y}=t_{x y}-\frac{1}{Q} \sum_{y=1}^{Q} t_{x y}, \bar{e}_{x y}=e_{x y}-\frac{1}{Q} \sum_{y=1}^{Q} e_{x y} .\right.
$$

Further processing of the formula can obtain

$$
e_{x y}=\left[\begin{array}{c}
\bar{t}_{x 1}, \ldots, \bar{t}_{x Q} \\
\bar{e}_{x 1}, \ldots, \bar{e}_{x Q}
\end{array}\right]=\bar{w}\left(\sum_{i=1}^{N} \delta_{x y} \lambda_{x}\right) .
$$

Ensure $m_{x y}$ and $m_{x y}$ projection error minimization; the motion parameter matrix and target intelligent tracking structure parameter matrix can be obtained. The process is shown in the formula:

$\min \sum_{x y}\left\|m_{x y}-m_{x y}^{\prime}\right\|^{2}=\min \sum_{x, y}\left\|m_{x y}-\left(w \sum_{i=1}^{N} \delta_{x y} \lambda_{x}\right)\right\|^{2}$

In this method, the motion parameter matrix is expressed by quaternion, and a $4 \times G$, where $g$ represents the number of frames of the overall moving image sequence. The quaternion method makes the rotation matrix free of singularity, and the orthogonality usually appears in the quaternion of four constants. Therefore, the quaternion method 
can avoid the complex orthogonal protection problem in the process of selecting matrix parameters. The quaternion method greatly improves the efficiency of solving the rotation matrix and the parameter matrix of three-dimensional motion results. The motion parameter matrix is initialized according to the motion parameters that can reflect the motion rigid region during athletes' movement, which can effectively describe the large-scale motion state of the human body. This method can solve the problem that the constraint information cannot be adjusted effectively due to the small time interval of the image sequence. The continuous detection of the direction of movement may be achieved by utilising the features of martial arts motions to determine the direction of movement in the video picture, and then using the moving target to locate the search window in the next frame of video image, and continuing this process. The method can extract the probability distribution of movement direction from the features of the video picture while detecting Wushu movement direction. The feature probability distribution of the video picture will vary as the detection target travels over time. The target may therefore be identified based on the change in the probability distribution of the action characteristic in the video picture. The specific flow of the algorithm is shown in Figure 6.

The whole flow of the algorithm is shown in the figure. The core parts of the algorithm are probability distribution of features, centroid of search window, and area of search window. Find the location of martial arts from the video image, which is called the average migration algorithm. Cluster analysis according to the characteristics of motion direction mainly includes the following steps:

(1) Set search window size.

(2) Determine the initial location of the search window.

(3) Calculate search window center position results.

(4) Repeat step (3) until convergence, and the moving distance of the center position of the video image is less than the previously assumed threshold.

For the discrete probability distribution image, the center position of the window is searched, and the zero order matrix can be calculated through the search window:

$$
Q_{00}=\sum_{A} \sum_{B} I(A, B) .
$$

Calculate the first-order matrix of $\mathrm{A}$ and $\mathrm{B}$ :

$$
\begin{aligned}
Q_{10} & =\sum_{A} \sum_{B} A I(A, B), \\
Q_{01} & =\sum_{A} \sum_{B} B I(A, B) .
\end{aligned}
$$

The core of the search window is

$$
\begin{gathered}
A=\frac{Q_{10}}{Q_{00}}, \\
B=\frac{Q_{01}}{Q_{00}} .
\end{gathered}
$$

In the above formula: $I(A, B)$ is the image pixel value at the point in the image, $A, B$ refers to the image pixel value at the point in the image, and the value range is taken in the whole search window. At the end of the search, the core location is the detected target's location at the current time. The continuous adaptive average migration algorithm is based on the dynamic probability distribution of the movement direction in the martial arts video image. In the continuous video image, it is easy for the change of the size and position of the martial arts target to cause the dynamic probability distribution to fluctuate greatly, but the size of the search window can be adjusted freely according to the probability distribution. Before each search, the initial value of the window is set to the current position and size of the motion direction. Because the search window detects near the area where the image target may appear, it can save a lot of search time and have good real-time performance.

\section{Analysis of Experimental Results}

In order to verify the feasibility of the application of the economic sports video analysis system designed above, opencv will be used to support video sequence image processing under the framework of MFC application program, and MATLAB software will be used for numerical calculation of moving target tracking algorithm and moving parameter calculation model. Taking diving Wushu as an example, the practical application effect in the process of moving target tracking will be tested. The configured test environment is as follows: hardware environment: $3.00 \mathrm{GHz}$ CPU, 160 GB memory. Software environment: Windows XP operating system, mysqlserver8.0.11 database, MATLAB 2013 b, opencv2.2. In order to verify the effectiveness of the improved target intelligent tracking algorithm proposed in this paper on moving objects, this section will experimentally verify and analyze the algorithm and compare it with other methods. The detailed configuration of the experimental computer is shown in Table 2 .

For the detection of target intelligent tracking method, in order to reduce the amount of calculation, we first gray-scale the color image and assume that pixels are independent of each other, and the Gaussian distribution corresponding to each pixel is also independent of the others. Next, experiments will be carried out on the impact of Gaussian distribution on the performance of the algorithm and the improvement of the detection effect of the target intelligent tracking algorithm. It can be seen from the figure that, with the increase of the number of Gaussian distributions in target intelligent tracking, the better the detection effect, the less noise, and the moving objects detected at the same time are more accurate, but through Table 3.

Take the automatic detection search window as the center, and register the six motion directions in the $5 * 5$ search window. If the automatic detection accuracy value is greater than the normal value, Step 4 needs to be carried out; if the automatic detection accuracy value is less than or equal to the normal value, Step 2 needs to be carried out, as shown in the figure. Assuming that the system automatically detects that the accuracy value of the East, West, North, and South 


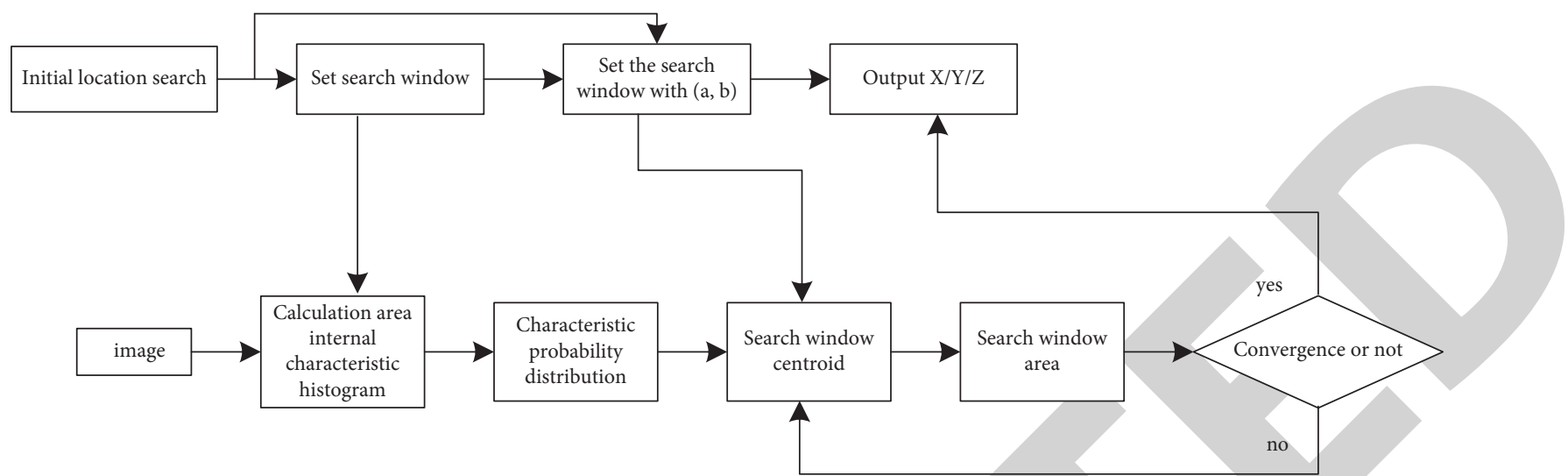

FIGURE 6: Information acquisition algorithm flow of target tracking video processing.

TABle 2: Experimental computer configuration.

\begin{tabular}{lc}
\hline Computer configuration & Detailed information \\
\hline CPU & Pentium $(R)$ dual-core E5300 2.6 GHz \\
Memory & DDR4 \\
Operating system & Windows \\
Software & Intelligent target tracking video \\
\hline
\end{tabular}

TABLE 3: Feedback running time of Wushu mixed action.

\begin{tabular}{lccc}
\hline Gaussian distribution $M$ & Video frames & Running time (s) & Average frame rate (frames/s) \\
\hline 2 & 90 & 42 & 2.45 \\
4 & 90 & 98 & 0.88 \\
6 & 90 & 150 & 0.65 \\
\hline
\end{tabular}

movement is greater than or equal to the accuracy value of the up and down movement, if the relevant value is within this range, the search window is $5^{*} 5$. According to the above contents, the search methods can be divided into two kinds: one is that the maximum value appears in the corner, so it is necessary to carry out the next search for these six points, as shown in Figure 5; the other is that if the maximum value appears on the horizontal line or vertical line; you also need to carry out the next search for these six points, as shown in Figure 7.

If the correlation value is less than the accuracy value of automatic detection of upward and downward movement of martial arts, the search method is as shown above, but the search window will become larger by $7^{*} 7$. When the search window is reduced to $3^{*} 3$, the distance between the six points in the window needs to be calculated. As shown in the figure, the maximum point of the correlation value is obtained by searching the center point within the range for normalization, which is the best registration point. Through the search of the registration point, the accuracy of automatic inspection can be improved, and the effectiveness is strong. On the experimental data, the running time of the Experimental Research Institute of image attitude parameter measurement method is compared between the traditional research method and the research method based on target intelligent tracking system. The results are shown in Figure 8.
It can be seen from the figure that when the number of experiments is 2 , the research time of the two methods is the same. The conventional method's research duration becomes unstable as the number of tests grows, fluctuating substantially up and down. Although the traditional method's research time is less than that of the method based on the target intelligent tracking system at several points during the experiment, the network is unstable, resulting in the traditional method's final research time being stable at about $760 \mathrm{~s}$; the research time based on the target intelligent tracking system method is relatively stable, and the fluctuation range is not large. Finally, using the target intelligent tracking system approach, the research time is steady at about 580 seconds. Therefore, the research time of targetbased intelligent tracking system method is shorter than that of traditional methods. The experimental research results of image attitude parameter measurement method based on traditional research methods and target intelligent tracking system are compared, and the results are shown in Figure 9.

It can be seen from the figure that the experimental time has a serious impact on the research effect of the research method, especially on the traditional method, which is $26 \%$ different from the maximum value and $20 \%$ different from the minimum value of the research method based on the target intelligent tracking system. The experimental time has little effect on the target-based intelligent tracking system's research method. In order to verify the time spent in the 


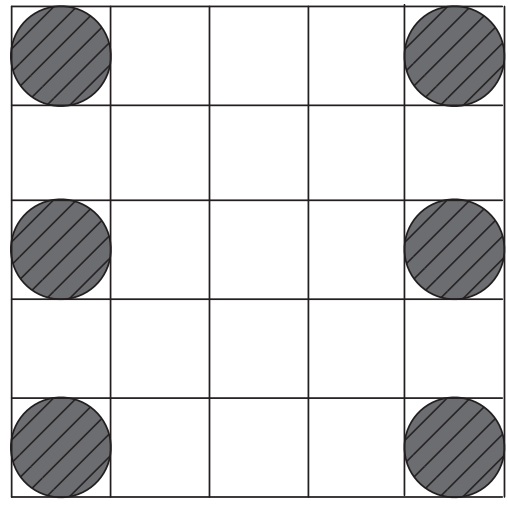

(a)

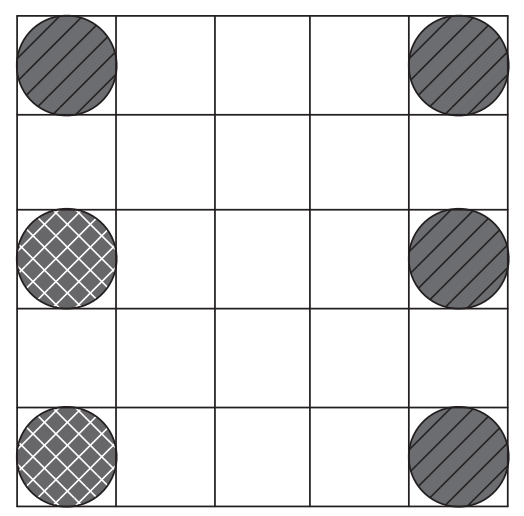

(b)

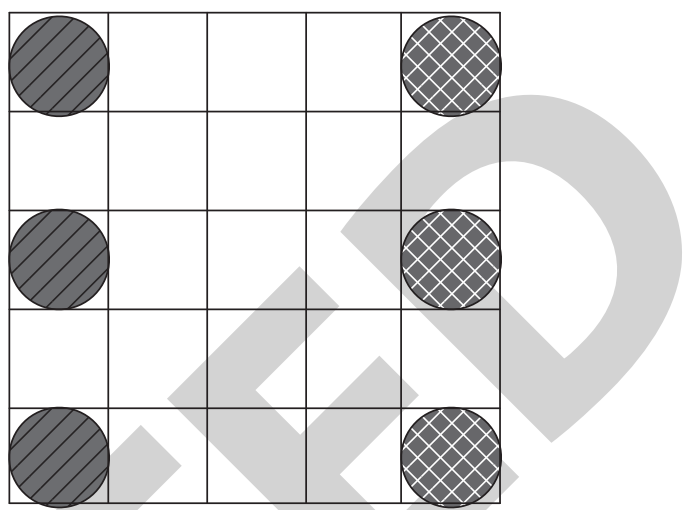

(c)

FIGURE 7: Search path.

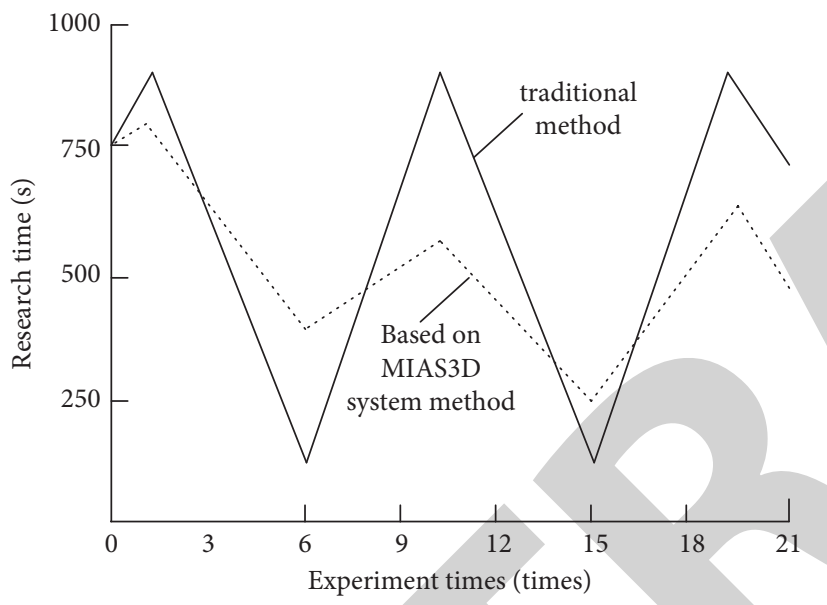

FIgURE 8: Time comparison results of two methods.

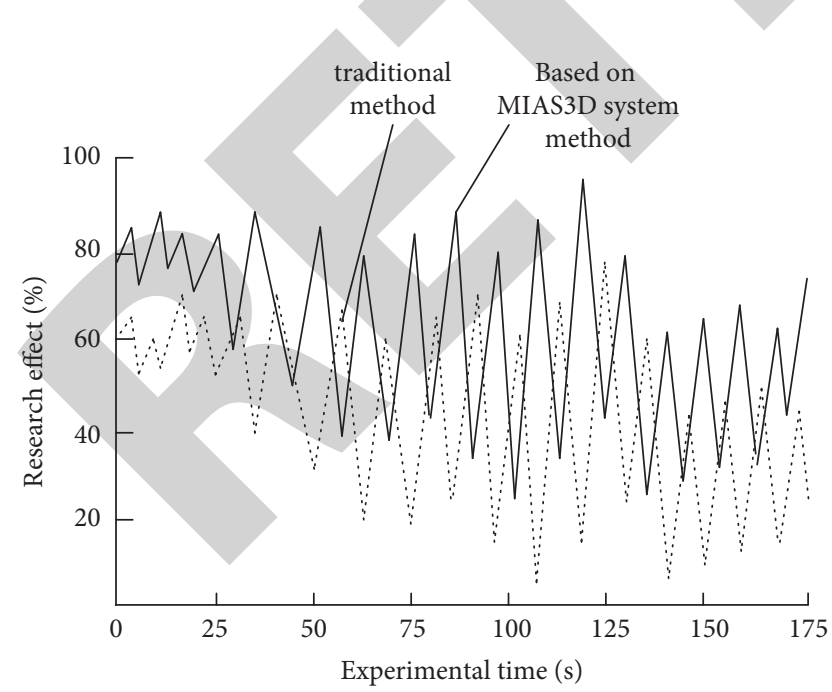

FIGURE 9: Comparative analysis of research effects of two methods.

automatic detection of the system, the kick direction in Wushu is taken as the detection standard, mainly including positive kick, bending kick, side kick, back kick, jumping kick, and running kick. The time consumed by the traditional system detection is compared with the time consumed
TABle 4: Comparison results of test time.

\begin{tabular}{lcc}
\hline Direction of motion & Traditional system (s) & Paper system (s) \\
\hline Positive kick & 60 & 36 \\
Bend kick & 68 & 28 \\
Side kick & 98 & 58 \\
Back kick & 88 & 45 \\
Jump kick & 87 & 26 \\
Running and kicking & 96 & 47 \\
\hline
\end{tabular}

by the motion direction motion feedback system based on video image, and the results are shown in Table 4.

It can be seen from the table that the time spent in using the traditional motion feedback system to detect the positive kick direction is $58 \mathrm{~s}$, which is longer than the time spent in the designed motion feedback system based on video image motion direction, while the time spent in detecting other kick directions is longer, which is far slower than the detection speed of the system designed in this paper.

\section{Conclusion}

Combined with the requirements of real-time scoring and optimization training of Wushu, based on previous research, and fully drawing on the design concept of computer-aided motion analysis system camas, the functions of three-dimensional human motion target detection and extraction, dynamic tracking, and behavior recognition are integrated. The designed video analysis system can meet the application needs of competitive level evaluation and training feedback according to the calculation and analysis of sports target parameters. The system can be self-contained or integrated into the system. It offers intelligent and data-based auxiliary support for Wushu sports training as the video analysis module of human motion target, avoiding the issue of misjudgment in Wushu sports events and realizing targeted and scientific sports training.

\section{Data Availability}

The data used to support the findings of this study are included within the article. 


\section{Conflicts of Interest}

The authors declare that they have no conflicts of interest.

\section{Acknowledgments}

This paper was supported by Beijing University of Physical Education, school independent subject "Research on the theory and index system construction of 24-style Taijiquan exercise load evaluation standard" (no. School 2020043).

\section{References}

[1] Z. Sutton, P. Willett, and Y. Bar-Shalom, "Target tracking applied to extraction of multiple evolving threats from a stream of surveillance data," IEEE Transactions on Computational Social Systems, vol. 8, no. 99, pp. 1-17, 2021.

[2] S. S. Moghaddasi and N. Faraji, "A hybrid algorithm based on particle filter and genetic algorithm for target tracking," Expert Systems with Applications, vol. 147, Article ID 113188, 2020.

[3] S. Tan, S. Zhong, and P. Chirarattananon, "A one-step visual-inertial ego-motion estimation using photometric feedback," IEEE/ASME Transactions on Mechatronics, vol. 27, no. 1, pp. 12-23, 2022.

[4] N. Sun, R. Sun, and S. Li, "Martial arts routine difficulty action technology VR image target real-time extraction simulation," IEEE Access, vol. 8, no. 99, p. 1, 2020.

[5] K. Ishac and D. Eager, "Evaluating martial arts punching kinematics using a vision and inertial sensing system," Sensors, vol. 21, no. 6, Article ID 1948, 2021.

[6] C. Zhao and B. Li, "Artificial intelligence auxiliary algorithm for Wushu routine competition decision based on feature fusion," Journal of Healthcare Engineering, vol. 2021, Article ID 1632393, 7 pages, 2021.

[7] D. Perpetuini, D. Cardone, C. Filippini, A. M. Chiarelli, and A. Merla, "A motion artifact correction procedure for fNIRS signals based on wavelet transform and infrared thermography video tracking," Sensors, vol. 21, no. 15, Article ID 5117, 2021.

[8] R. Yuan, Z. Zhang, and P. Song, "Construction of virtual video scene and its visualization during sports training," IEEE Access, vol. 8, no. 99, p. 1, 2020.

[9] J. L. Felipe, J. Garcia-Unanue, D. Viejo-Romero, A. Navandar, and J. Sánchez-Sánchez, "Validation of a video-based performance analysis system (mediacoach) to analyze the physical demands during matches in LaLiga," Sensors, vol. 19, no. 19, Article ID 4113, 2019.

[10] J. Ma, "Research on sports video image based on clustering extraction," Mathematical Problems in Engineering, vol. 2021, Article ID 9996782, 9 pages, 2021.

[11] L. Shidong, T.-H. Kuo, M. ul Islam, M. Talha, S. A. Lone, and S.-Y. Wei, "The impact of sports activities on economic survival, social life of women, and what do big data analysis reveals about social life of sports women," Revista de Psicologia del Deporte, vol. 30, no. 3, pp. 229-241, 2021.

[12] S. Chen and G. Zhao, "High-performance server-based live streaming transmission optimization for sports events in smart cities," Mobile Information Systems, vol. 2021, Article ID 9958703, 7 pages, 2021.

[13] B. Pueo, J. J. Lopez, and J. M. Jimenez-Olmedo, “Audio-based system for automatic measurement of jump height in sports science," Sensors, vol. 19, no. 11, Article ID 2543, 2019.
[14] L. Zhu, "Computer vision-driven evaluation system for assisted decision-making in sports training," Wireless Communications and Mobile Computing, vol. 2021, Article ID 1865538, 7 pages, 2021.

[15] M. A. Lopez-Gordo, N. Kohlmorgen, and C. Morillas, "Performance prediction at single-action level to a firstperson shooter video game," Virtual Reality, vol. 23, no. 1, pp. 1-13, 2020.

[16] S. Yang, "Construction of video courses of physical education and health education in colleges and universities under the MOOC platform," Mobile Information Systems, vol. 2021, Article ID 9925838, 8 pages, 2021.

[17] S. Tan, S. Zhong, and P. Chirarattananon, "A one-step visualinertial ego-motion estimation using photometric feedback," IEEE, vol. 2021, no. 99, p. 1, 2019.

[18] L. I. U. Guanyang and X. G, "Haptic based teleoperation with master-slave motion mapping and haptic rendering for space exploration," Chinese Journal of Aeronautics, vol. 32, pp. 181-194, 2019.

[19] H. U. Dike and Y. Zhou, "A robust quadruplet and faster region-based $\mathrm{CNN}$ for UAV video-based multiple object tracking in crowded environment," Electronics, vol. 10, no. 7, p. 795, 2021.

[20] M. R. Marshall, D. Hellfeld, and T. Joshi, “Three-dimensional object tracking in panoramic video and LiDAR for radiological source-object attribution and improved source detection," IEEE Transactions on Nuclear Science, vol. 15, no. 99, p. $1,2020$.

[21] U. Srilakshmi, N. Veeraiah, Y. Alotaibi, S. A. Alghamdi, O. I. Khalaf, and B. V. Subbayamma, "An improved hybrid secure multipath routing protocol for MANET," IEEE Access, vol. 9, pp. 163043-163053, 2021. 